\title{
Neurocan is a New Substrate for the ADAMTS12 Metalloprotease: Potential Implications in Neuropathies
}

\author{
Tania Fontanila,b,c,d Yamina Mohamedia, Angela Moncada-Pazos ${ }^{a, b, e}$ \\ Teresa Cobo c,f José A. Vegag,h Juan Luis Cobog,i Olivia García-Suárez ${ }^{g}$ \\ Juan Cobo ${ }^{c, f}$ Álvaro J. Obaya ${ }^{\mathrm{b}, j}$ Santiago Cal ${ }^{\mathrm{a}, \mathrm{b}}$
}

\begin{abstract}
aDepartamento de Bioquímica y Biología Molecular, Universidad de Oviedo, Oviedo, Spain, bInstituto Universitario de Oncología, IUOPA, Universidad de Oviedo, Oviedo, Spain, Instituto Asturiano de Odontología, Oviedo, Spain, dDepartamento de Investigación, Clínica Ordóñez, Oviedo, Spain, ePresent address: Orbit Discovery Ltd., Oxford, United Kingdom, 'Departamento de Cirugía y Especialiades Médico-Quirúrgicas, Universidad de Oviedo, Oviedo, Spain, 9Departamento de Morfología y Biología Celular, Facultad de Medicina, Universidad de Oviedo, hFacultad de Ciencias de la Salud, Universidad Autónoma de Chile, Santiago, Chile, iServicio de Cirugía Maxilofacial, Hospital Universitario Central de Asturias (HUCA), Oviedo, Spain, jDepartamento de Biología Funcional, Área de Fisiología, Universidad de Oviedo, Oviedo, Spain
\end{abstract}

\section{Key Words}

Metalloprotease $\cdot$ Extracellular matrix $・$ Neurocan $\cdot$ ADAMTS $・$ Neurocanase

\begin{abstract}
Background/Aims: The composition of the extracellular matrix (ECM) in the central nervous system (CNS) has several features that make it unique. For instance, it is remarkable for the presence of proteoglycans such as versican, brevican, and neurocan, some of which have been identified as substrates of different members of the ADAMTS family of secreted metalloproteases. Previous studies have associated ADAMTSs with the repair of the CNS, including recovery following degradation of glial scar tissue and the stimulation of axonal growth after brain injury. However, the involvement of ADAMTSs in diseases of the CNS is complex and not understood fully, and a current challenge is unraveling the precise roles of these metalloproteases in the brain. Methods: ADAMTS12 and neurocan gene expression was examined by quantitative PCR. Western blot analysis was employed to detect ADAMTS12 and neurocan protein expression in cell lines, and immunostaining techniques were used to detect neurocan in mouse brain tissues. Neurocan cleavage using recombinant ADAMTS1, ADAMTS4, ADAMTS5, and ADAMTS12 metalloproteases was evaluated by western blotting. Cell adhesion and migration were assessed using uncoated culture dishes or dishes coated with Matrigel or ECM components. Results: We identified neurocan as a novel component of brain ECM that can be cleaved by ADAMTS12. In addition, we showed that neurocan


cleavage by ADAMTS12 altered the adhesive properties of the human neuroglioma $\mathrm{H} 4$ cell line. Moreover, immunohistochemical analysis of Adamts12-deficient mice revealed the significant accumulation of neurocan in the brain of neonatal mice. Conclusion: Overall, our results suggest that ADAMTS12 could be involved in the repair of the CNS through its ability to degrade neurocan. Moreover, it can be inferred that alterations in neurocan degradation processes could be associated with the pathogenesis of neurological disorders.

(c) 2019 The Author(s). Published by Cell Physiol Biochem Press GmbH\&Co. KG

\section{Introduction}

The composition of the extracellular matrix (ECM) is specific for each tissue. In particular, the ECM in the central nervous system (CNS) shows several aspects that make it unique compared with the ECM of other tissues [1]. For instance, low levels of collagen and other fibril-forming molecules are present in the ECM of the CNS. In contrast, its main components include hyaluronan, glycoproteins such as tenascin, and hyalectans [2]. Hyalectans are a subfamily of proteoglycans characterized by the presence of chondroitin sulfate-type glycosaminoglycan groups within their structure. These groups are important for binding to hyaluronic acid and other molecules of the ECM to form a three-dimensional network that has important functions in the homeostasis of nervous tissue [3]. Members of the hyalectan family include aggrecan, versican, brevican, and neurocan [4], of which the last three play vital roles in the maintenance of brain function. For instance, following injury in the CNS, scar tissue is mainly formed by this type of chondroitin sulfate proteoglycan. However, proteoglycans also inhibit repair mechanisms [5-8], indicating that for the full restoration of injured CNS tissue, mechanisms involving chondroitin sulfate degradation and elimination are needed in order to reestablish normal homeostasis.

Extracellular proteases are key components of these processes due to their ability to degrade hyalectans selectively. This capacity makes these enzymes essential factors to regulate the balance between proteoglycan formation and degradation during tissue repair. The proteases mainly involved in these processes are matrix metalloproteinases and A disintegrin and metalloproteinases with thrombospondin motifs (ADAMTSs) [9]. ADAMTSs are secreted enzymes studied widely in relation to fundamental processes such as tissue morphogenesis and inflammation, or in the circulatory system [3, 10]. In humans, 19 ADAMTSs have been identified, and they are arranged in subcategories mainly according to their structure and known substrates. The ability of ADAMTSs to degrade ECM components has been shown widely, especially for three of the four hyalectans: aggrecan, versican, and brevican [11-13]. The remaining member of the hyalectan family, neurocan, is also cleaved in various processes involving nervous tissue [14,15], although the mechanism governing this degradation remains to be characterized fully. Neurocan is expressed at a low level in the adult mouse brain in normal conditions [16]. However, its expression is increased considerably after brain damage and its participation in retaining repairing cells within the damaged tissue is, in turn, responsible for inducing inflammation [15, 17]. To avoid prolonging this response, the regulated degradation and further elimination of neurocan are necessary.

Among ADAMTSs, ADAMTS12 is known to participate in and be necessary for inflammation, since mice deprived of this protease show prolonged inflammation in models of pancreatitis, colitis, and lipopolysaccharide-induced inflammation [18]. In the present study, we show that neurocan is cleaved by ADAMTS12 in vitro and in vivo. Furthermore, neurocan degradation by ADAMTS12 results in changes in the adhesion and migration profiles of the human neuroglioma $\mathrm{H} 4$ cell line. The adhesion of the human microglial SIM-A9 cell line, in which endogenous neurocan expression can be detected, is also altered upon RNA interference (RNAi) to hamper ADAMTS12 gene expression. Taken together, our results suggest that alterations in neurocan degradation by ADAMTS12 could affect the normal 


\section{Cellular Physiology Cell Physiol Biochem 2019;52:1003-1016 \\ \begin{tabular}{ll|l} 
and Biochemistry & $\begin{array}{l}\text { DOl: 10.33594/000000069 } \\
\text { Published online: } 13 \text { April 2019 }\end{array}$ & $\begin{array}{l}\text { O } 2019 \text { The Author(s). Published by } \\
\text { Cell Physiol Biochem Press GmbH\&Co. KG }\end{array}$ \\
\cline { 2 - 3 } &
\end{tabular} \\ Fontanil et al.: Neurocan Cleavage by ADAMTS12}

function of the ECM in the brain, which could shed light on the molecular bases underlying neurological disorders.

\section{Materials and Methods}

Cell lines, cell culture conditions, and transfection

The H4 cell line derived from a human neuroglioma was a generous gift from Dr. Alberto Lleó (Servicio de Neurología, Hospital Santa Cruz y San Pablo, Barcelona). The mouse microglial SIM-A9 cell line was purchased from Bio-Cat. The cells were cultured routinely at $37^{\circ} \mathrm{C}$ in a $5 \% \mathrm{CO}_{2}$ atmosphere in basal Dulbecco's modified Eagle's medium supplemented with $10 \%$ fetal bovine serum, $50 \mu \mathrm{g} / \mathrm{mL}$ streptomycin, and $100 \mathrm{U} /$ $\mathrm{mL}$ penicillin (Life Technologies). ADAMTS12 recombinant protein was obtained as described previously [19]. To perform RNAi of the ADAMTS12 gene in SIM-A9 cells, the Adamts12 Mouse shRNA Plasmid (Locus ID: 239337) from OriGene was employed according to the manufacturer's recommendations.

\section{Expression analysis by PCR amplification}

Quantitative PCR (qPCR) amplification was used to study ADAMTS12 expression levels in a set of 48 PCR-ready, first-strand cDNA samples from mouse CNS tissues (Mouse Developmental Tissue qPCR Panel; OriGene) at 5 different developmental stages: 3 embryonic, 1 postnatal, and 1 adult. Analysis was carried out following the manufacturer's instructions on an ABI Prism 7300 sequence detector (Applied Biosystems). To perform neurocan amplification, qPCR reactions were carried out using Fast SYBR Green technology (Sigma-Aldrich) and the following oligonucleotides: forward 5'-CAACACAGGACTGCAATATG-3' and reverse $5^{\prime}$-TATAGGGTAGGTTGTAGTTGC- $3^{\prime}$. Gene expression was normalized to the mouse GAPDH gene (forward 5'-TGCCACTGCAAATGGCAGCCC-3'; reverse 5'-GTGCCAGCCTCGTCCCGTAGA-3'). To amplify the murine ADAMTS12 gene, we employed TaqMan MM00615603_M1 FAM-MGB from Thermo Fisher Scientific. GAPDH amplification was also employed to normalize gene expression using the 4352339E NM_008084.2 VIC probe from Applied Biosystems. Relative expression was calculated as $R Q=2^{-\Delta \Delta c t}$.

\section{Western blot analysis}

For western blot analysis, cell extracts were resolved by $10 \%$ sodium dodecyl sulfate (SDS)polyacrylamide gel electrophoresis and transferred to a polyvinylidene difluoride membrane (Millipore). An anti-ADAMTS12 antibody was purchased from Proteintech (Ref. 24934-1-AP). Neurocan was detected using a mouse/rat anti-neurocan antibody from R\&D Systems (Ref. AF5800). When indicated, the loading control was examined using an anti- $\alpha$-actin antibody from Sigma-Aldrich (Ref. SAB5500001). Immunoreactive proteins were visualized using horseradish peroxidase (HRP)-labeled anti-rabbit or anti-mouse secondary antibodies and the ECL detection system (Pierce).

\section{Neurocan immunostaining in the CNS}

A collection of brain tissues from day 14 embryos and newborn mice was employed to evaluate neurocan levels. The slides were processed for indirect peroxidase immunohistochemistry in the following way: the sections were deparaffinized and rehydrated, and then rinsed in phosphate-buffered saline (PBS) containing 1\% Tween-20. For neurocan detection (anti-neurocan antibody from R\&D Systems, Ref. AF5800), the sections were heated in high pH Envision FLEX target retrieval solution at $80^{\circ} \mathrm{C}$ for $20 \mathrm{~min}$ and incubated for $20 \mathrm{~min}$ at room temperature in the same solution. Endogenous peroxidase activity $\left(3 \% \mathrm{H}_{2} \mathrm{O}_{2}\right)$ and non-specific binding (33\% fetal calf serum) were blocked and the sections were incubated overnight at $4{ }^{\circ} \mathrm{C}$ with a primary antibody (1:100 dilution) as described above. A secondary antibody labeled with polymer-HRP (DAKO) was used. 3, 3'-Diaminobenzidine was employed as a chromogen. Selected slides were counterstained with hematoxylin. 


\section{Cellular Physiology Cell Physiol Biochem 2019;52:1003-1016 \\ \begin{tabular}{ll|l} 
and Biochemistry & Dublished online: 13 April 2019 & $\begin{array}{l}\text { C) } 2019 \text { The Author(s). Published by } \\
\text { Cell Physiol Biochem Press GmbH\&Co. KG }\end{array}$
\end{tabular} \\ Fontanil et al.: Neurocan Cleavage by ADAMTS12}

\section{Enzymatic assay}

Neurocan degradation $(0.5 \mu \mathrm{g})$ was performed using recombinant mouse neurocan protein (R\&D Systems, Ref. 5800-NC) in $30 \mu \mathrm{L}$ reaction buffer ( $50 \mathrm{mM}$ Tris- $\mathrm{HCl}, 150 \mathrm{mM} \mathrm{NaCl}, 10 \mathrm{mM} \mathrm{CaCl}, 0.05 \%$ Brij 35, pH 8.5). Recombinant ADAMTS1 (Ref. 2197-AD), ADAMTS4 (Ref. 4307-AD), or ADAMTS5 (Ref. 2198-AD), purchased from R\&D Systems, was added at $50 \mathrm{nM}$. Recombinant ADAMTS12 was obtained as described previously [19]. The reactions were allowed to proceed at $37^{\circ} \mathrm{C}$ for the indicated times, $1 \mathrm{mU}$ chondroitinase ABC (Sigma-Aldrich) was added for $30 \mathrm{~min}$, and the reactions were stopped by the addition of a reducing SDS-sample buffer containing 20 mM EDTA. Digestion products were visualized by western blot analysis.

\section{Adhesion assay}

The adhesion of H4 cells was evaluated using uncoated culture dishes (Ibidi, Ref. 81151). To examine whether intact neurocan or neurocan cleaved by ADAMTS12 modified the adhesive properties of this cell line, the culture dishes were coated with undigested neurocan protein in increasing quantities $(0.2,1.0$, and $2.0 \mu \mathrm{g} / \mathrm{mL}$ ) or neurocan digested previously with ADAMTS12. When indicated, the dishes were also coated with recombinant ADAMTS12 $(30 \mu \mathrm{g} / \mathrm{mL}$ ) or type IV collagen (Sigma-Aldrich, $15 \mu \mathrm{g} / \mathrm{mL}$ ). Uncoated wells were employed as a negative control. Once the wells were prepared, $1.2 \times 10^{5} \mathrm{H} 4 \mathrm{cells} / \mathrm{mL}$ were seeded and allowed to adhere in an incubator at $37{ }^{\circ} \mathrm{C}$ and $5 \% \mathrm{CO}_{2}$ for $1 \mathrm{~h}$. Non-adhered cells were removed by washing three times with PBS, and the remaining cells were fixed and stained with a cell staining solution. Images were captured with a Zeiss Axiovert $200 \mathrm{M}$ type and analyzed using Image software. The adhesion of SIM-A9 cells was also examined. The procedure was basically as described above, but in this case, the culture dishes were coated with Matrigel (BD Biosciences, Ref. 356230).

\section{Migration assay}

To evaluate the migration of H4 cells, we coated Ibidi plates (Ibidi, Ref. 81151) with ADAMTS12 (30 $\mu \mathrm{g} / \mathrm{mL})$, recombinant human neurocan $(0.2 \mu \mathrm{g} / \mathrm{mL})$, or neurocan digested with ADAMTS12. We seeded $6.0 \times 10^{5}$ cells into the wells of the inserts (Ibidi, Ref. 80209) and the cells were allowed to adhere for 1 h. Then, the inserts were removed, introducing a cell-free zone with a width of $500 \mu \mathrm{m}$. We added $2 \mathrm{~mL}$ culture medium, and cell migration was followed by time-lapse imaging for $24 \mathrm{~h}$ using a Zeiss Axiovert 200 microscope coupled to an XL-multi S1 incubator. Movies and images were taken using AxioVision software release 4.7.2, and migration areas at different time points were calculated using Image J software.

\section{Statistical analysis}

Data were analyzed using GraphPad software and are presented as the mean \pm standard error. Significant differences were determined with Student's $t$ test or one-way analysis of variance followed by Tukey's multiple comparison test. $P$-values less than 0.05 were considered statistically significant.

\section{Results}

\section{ADAMTS12 and neurocan expression in mouse CNS}

Members of the ADAMTS family of metalloproteases participate in the regulation of neuronal plasticity and in repair mechanisms after brain injury [20]. Among them, ADAMTS12 is implicated in inflammation and has been genetically linked to neuropathologies such as schizophrenia [21]. As a first approach to unravel the role of ADAMTS12 in the CNS, we examined its expression by qPCR using a commercial set of 48 PCR-ready, first-strand cDNA samples obtained from mouse brain tissues at different developmental stages (Fig. 1). ADAMTS12 was detected at different expression levels in the three different embryonic developmental stages analyzed (embryonic days 13,15, and 18), but its expression was reduced at postnatal day 7 . In tissue from 5 -week-old mice, ADAMTS12 expression remained at low level and it was mainly detected in the olfactory bulb and striatum. qPCR analysis also revealed that neurocan was expressed in the three embryonic developmental stages, but it was poorly detected in postnatal and adult mouse brain (Fig. 1). To gain more information about neurocan and ADAMTSs expression in the brain, we accessed the Allen Brain Atlas database (http://www.brain-map.org). According to this database, neurocan was detected 


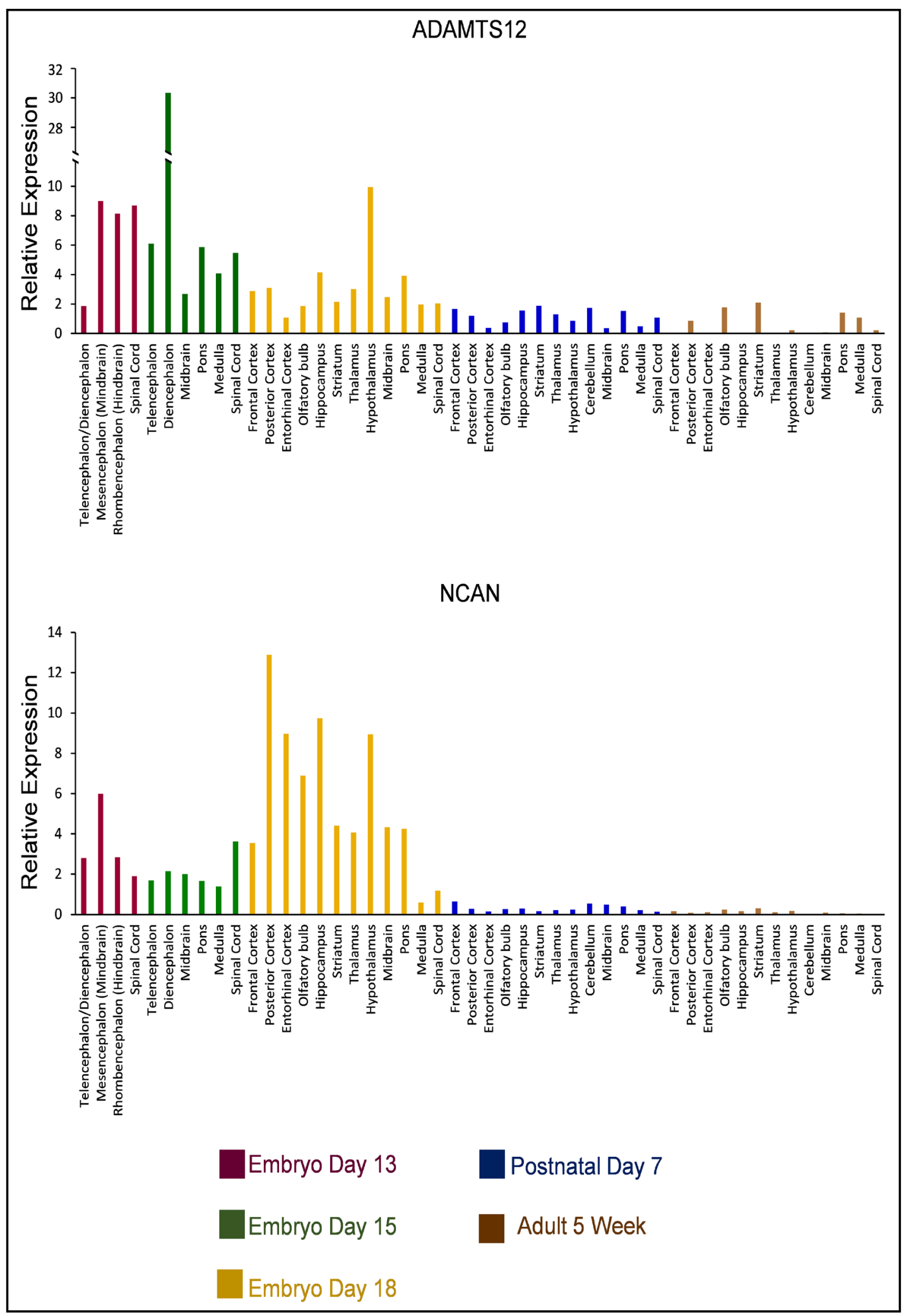

Fig. 1. Analysis of ADAMTS12 and neurocan (NCAN) expression by qPCR using a set of 48 commercially available PCR-ready, first strand cDNAs obtained from mouse tissues at different developmental stages, as indicated. GAPDH amplification was performed to normalize the obtained values. 
Fig.2. Neurocan cleavage by ADAMTSs. A. In vitro digestion of murine neurocan by ADAMTSs. Neurocan was incubated with ADAMTS1 $($ NCAN + ADAMTS1), ADAMTS4 (NCAN + ADAMTS4), ADAMTS5 (NCAN + ADAMTS5), or ADAMTS12 (NCAN + ADAMTS12) for 16 h. NCAN indicates neurocan incubated alone (without metalloproteases, control). Intervening irrelevant lanes are not shown. The presence of the proteolytic products of ADAMTS12 activity is indicated with arrows. Molecular weight markers are indicated on the left (kDa) B. Neurocan was incubated in the presence $(+)$ or absence $(-)$ of ADAMTS12 for $0,4,8$, and 12 h. Molecular weight markers are indicated on the left $(\mathrm{kDa})$. C. Analysis of the expression of ADAMTS12 (top) and neurocan (NCAN, bottom) in control and RNAi (shADAMTS12) SIM-A9 cells. Samples were loaded in triplicate. Molecular weight markers are indicated on the left $(\mathrm{kDa}) . \beta$-Actin was employed as a loading control.

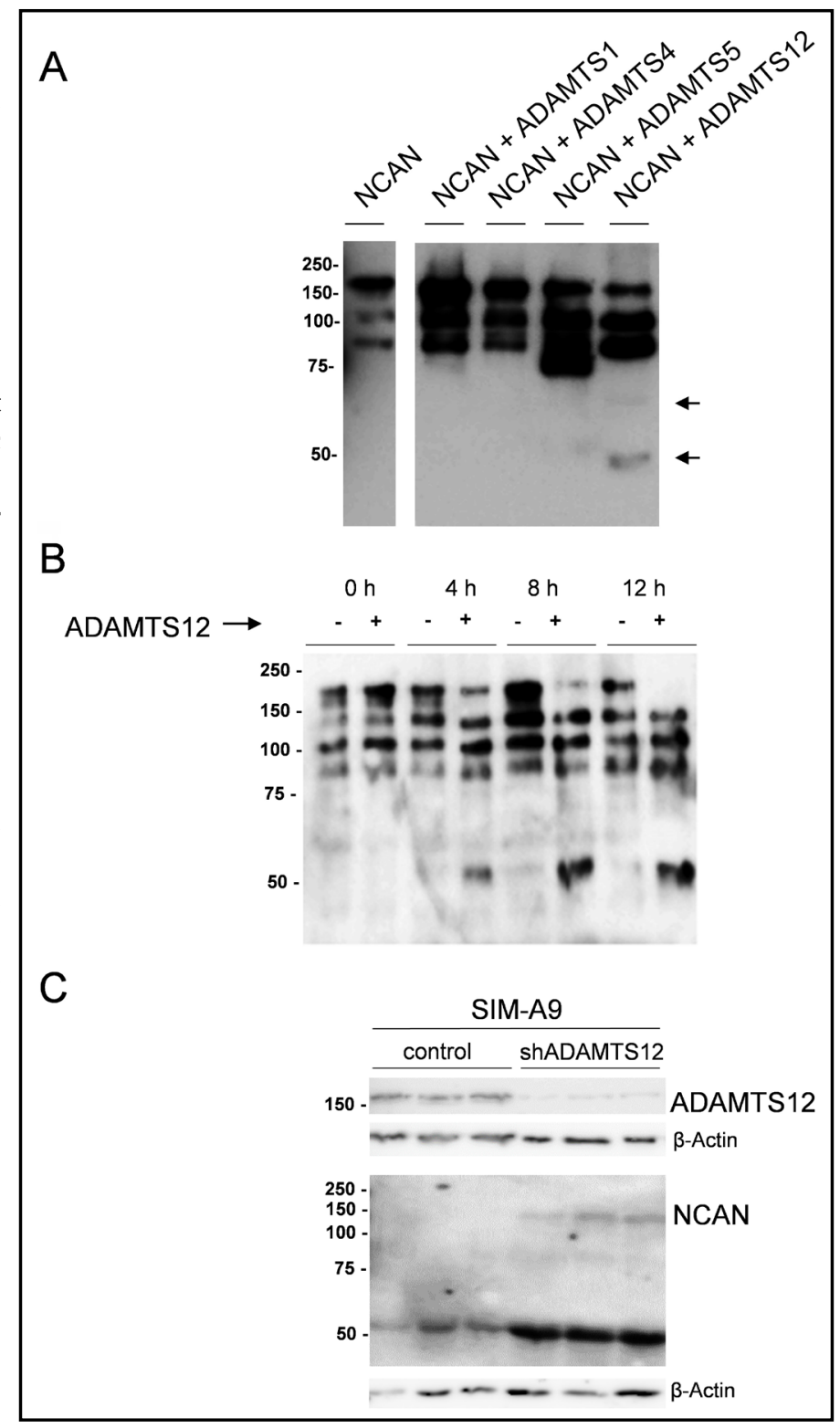

in the olfactory area, among other areas, in adult mice, and ADAMTS12 was also detected in the olfactory area (Supplementary Fig. 1 - for all supplemental material see www. cellphysiolbiochem.com). Moreover, neurocan expression was mainly detected in human neonate and infant brains, but its expression was drastically decreased in childhood and adult brains according to data available at www.szdb.org (A Database for Schizophrenia Genetic Research) [22] (Supplementary Fig. 2).

\section{Neurocan cleavage by ADAMTSs}

Expression data analysis prompted us to consider neurocan as a potential substrate for ADAMTS12. To evaluate that possibility, we analyzed the ability of ADAMTS12 to cleave neurocan in vitro, as well as that of three well-known hyalectanases (ADAMTS1, ADAMTS4, and ADAMTS5). The results shown in Fig. 2A indicate that ADAMTS5 and ADAMTS12 could cleave murine neurocan, but showed notable differences. That is, the digestion pattern for ADAMTS12 showed a main specific proteolytic product of approximately $50 \mathrm{kDa}$, which was absent in the cleavage reaction mediated by ADAMTS5. These data could indicate that 
ADAMTS12 has a higher specificity toward neurocan degradation than the other ADAMTSs assayed. It is also worth mentioning that neither ADAMTS1 nor ADAMTS4 showed a strong ability to cleave neurocan under the same experimental conditions. Interestingly ADAMTS1, ADAMTS4, and ADAMTS5 share the ability to digest not only aggrecan but also other hyalectans such as versican and brevican [23].

To ascertain the capacity of ADAMTS12 to degrade neurocan, we also performed a timecourse experiment (Fig. 2B). As early as $4 \mathrm{~h}$, the 50 -kDa band was detected when neurocan was incubated in the presence of ADAMTS12, but it was not observed in its absence. After $12 \mathrm{~h}$, the band corresponding to full-length neurocan was not detected, whereas the 50-kDa band remained as the main degradation product, as demonstrated by the increased intensity of the immunoreactive signal.

Next, we examined whether cleavage of neurocan could take place in cells in the presence of ADAMTS12. To this end, we employed the mouse microglial SIM-A9 cell line, as detectable amounts of ADAMTS12 can be visualized by western blotting (Fig. 2C). An anti-neurocan antibody also detected a unique $50-\mathrm{kDa}$ band that could correspond to a cleaved form of neurocan. Analysis of cell extracts from SIM-A9 cells in which ADAMTS12 expression was knocked down by RNAi revealed the presence of a 150-kDa band of neurocan. These data do not exclude the possibility that other proteases can cleave neurocan in SIM-A9 cells, but the presence of the 150-kDa band from neurocan following inhibition of ADAMTS12 strongly suggests that neurocan is a substrate of ADAMTS12 in vitro.

\section{Cleaved neurocan increases the adhesion of the H4 neuroglioma cell line}

Neurocan plays an important role in the healing process that follows any brain injury. In fact, neurocan provides physical support for cells to anchor to, and thus, to begin the process of tissue repair. Therefore, we analyzed the effect of neurocan cleavage by ADAMTS12 on the adhesion of H4 cells, a neuroglioma-derived cell line. According to the data available at the Cancer Cell Line Encyclopedia (https://portals.broadinstitute.org/ccle), H4 cells do not express neurocan, but they do express ADAMTS12. To examine cell adhesion, we coated culture dishes with neurocan, ADAMTS12, or neurocan cleaved by ADAMTS12. As a negative control, we employed uncoated dishes, whereas dishes coated with type IV collagen were used as a positive control. As shown in Fig. 3A, collagen type IV supported cell adhesion and it did so with much more efficiency than neurocan alone at $0.2 \mu \mathrm{g} / \mathrm{mL}$ (average of $99.8 \mathrm{vs}$. 17.2 cells per field, respectively). Dishes coated with ADAMTS12 alone showed an average of 21.16 cells per field, and a similar number of cells were found attached to dishes coated with neurocan digested by ADAMTS12 (21.33 cells per field). However, we observed differences between those conditions when the concentrations of neurocan and ADAMTS12-cleaved neurocan used for dish coating were increased. In fact, at $1 \mu \mathrm{g} / \mathrm{mL}$ neurocan, the average number of cells per field increased to 28.6 vs. 44.3 in dishes coated with ADAMTS12-cleaved neurocan. At $2 \mu \mathrm{g} / \mathrm{mL}$, the differences were even larger and were raised to $56.83 v s$. 23.5 cells per field for each respective condition.

We also wanted to examine whether the adhesive properties of SIM-A9 cells were altered upon RNAi of ADAMTS12 expression. To this end, we examined the ability of these cells to bind to Matrigel, which is commonly used as a basement membrane rich in ECM components. As shown in Fig. 3B, a reduction in the expression levels of ADAMTS12 (shADAMTS12) led to a reduction in the number of cells attached to Matrigel with respect to control SIM-A9 cells, which were transfected with an empty vector (20 vs. 145 cells per field, respectively).

Overall, these data allow us to suggest that the adhesion profile of neural cells can be altered upon cleavage of neurocan or the reduction of ADAMTS12 expression.

Neurocan increases the migration capacity of H4 cells, but this property is hampered by ADAMTS12

After injury, cells change morphology and acquire properties that facilitate the repair process. Thus, cells should become attached to the injured area, but they also need to migrate in order to initiate the repair process. For that reason, once we evaluated the potential 


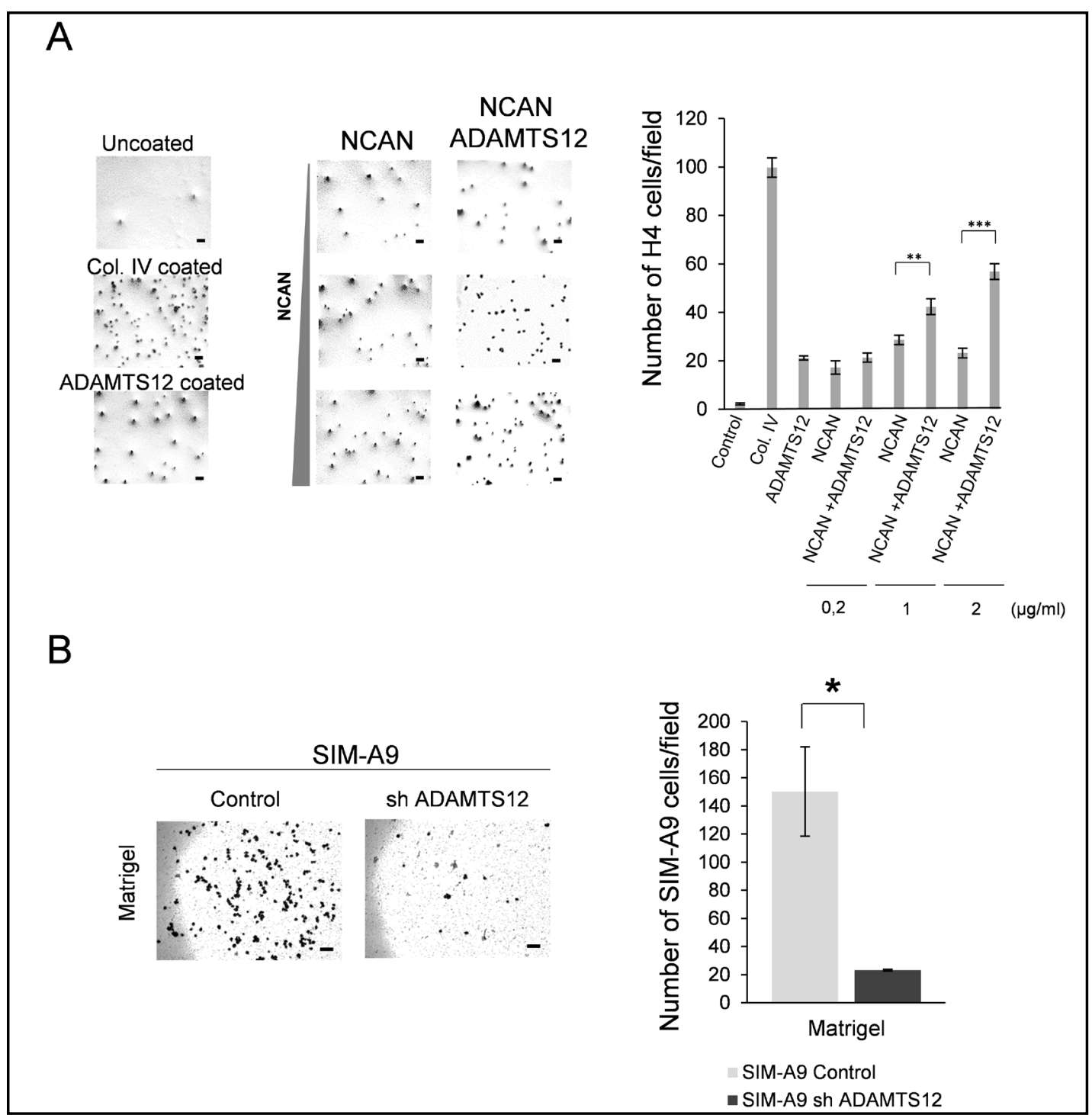

Fig. 3. The presence of neurocan and ADAMTS12 modifies the adhesion profile of human neuroglioma H4 cells and mouse microglial SIM-A9 cells. A. Left, representative images of $\mathrm{H} 4$ cells adhered to the indicated molecules following incubation for $1 \mathrm{~h}$. Neurocan (NCAN) was dispensed in increasing amounts $(0.2,1.0$, and $2.0 \mu \mathrm{g} / \mathrm{mL}$ ), and the same increasing amounts of neurocan were digested with ADAMTS12 (NCAN + ADAMTS12). Right, graphical representation of the number of adherent cells per field. Uncoated dishes were used as a negative control, and dishes coated with type IV collagen (col. IV) were used as a positive control. B. Left, representative images of control and ADAMTS12 RNAi (shADAMTS12) SIM-A9 cells adhered to Matrigel. Right, graphical representation of the number of cells per field. ${ }^{*} \mathrm{p}<0.05,{ }^{* *} \mathrm{p}<0.01,{ }^{* * *} \mathrm{p}<0.005$. Scale bar: $100 \mu \mathrm{m}$.

changes in the adhesion profile of cells following proteolytic cleavage of neurocan, we examined whether this cleavage also affects their migration. For that purpose, we performed a cell migration assay in which the cells were seeded on dishes coated with neurocan, ADAMTS12, or neurocan digested with ADAMTS12. As a control we used standard culture surfaces. H4 cells were seeded close to confluency and allowed to create two clearly visible edges separated by a gap of $500 \mu \mathrm{m}$.

To monitor cell migration, images were taken at different times, and after $24 \mathrm{~h}$, the area covered in each condition was measured (Fig. 4). The results revealed that coating the dishes with non-digested neurocan increased cell motility compared to ADAMTS12 and the control 
condition. The percentage of area covered was close to $100 \%$ for the dish coated with nondigested neurocan, $74 \%$ for its cleaved form, $62 \%$ for ADAMTS12-coated dishes, and $66 \%$ for the control condition. However, it is noteworthy that employing neurocan cleaved by ADAMTS12 as support for migration reduced the ability of $\mathrm{H} 4$ cells to migrate as compared with intact neurocan. These results suggest a role for neurocan in facilitating cell migration and also the requirement for its degradation in order to modulate cell motility and thus compromising the ability of cells to remain in injured tissue to initiate its restoration.

\section{Neurocan accumulates in newborn Adamts12-deficient mice}

Our previous results indicating the degradation of neurocan by ADAMTS12 prompted us to examine potential differences in neurocan localization between wild-type and Adamts12deficient mice. For that purpose, and bearing in mind that ADAMTS12 is preferentially expressed in embryonic tissues, we analyzed neurocan expression in day 14 embryonic mice and newborn mice. We first observed the absence of neurocan immunostaining in day 14 wild-type and Adamts12-deficient embryos. When neonatal mice were tested, neurocan expression was not detected anywhere in the CNS of wild-type mice, neither in the brain nor in the spinal cord (Fig. 5A-C). Conversely, in Adamts12-deficient mice (Fig. 5D-H), diffuse immunostaining was observed in the brain, especially in the olfactory bulb (internal and external plexiform strata) and hypothalamus, as well as in the spinal ganglia (Fig. 5D). Overall, these results indicate that neurocan levels are increased in the absence of ADAMTS12 and allow us to suggest that this metalloprotease acts as a neurocan-cleaving enzyme.

Fig. 4. Increased migration of $\mathrm{H} 4$ cells on neurocan. A. H4 cells were allowed to migrate in wells coated with ADAMTS12, neurocan (NCAN), or neurocan cleaved by ADAMTS12 (NCAN + ADAMTS12). A standard culture dish was employed as a control. Representative pictures of starting $(\mathrm{t}=0 \mathrm{~h})$ and final $(\mathrm{t}=24 \mathrm{~h})$ times are included. The starting points are indicated with thin lines, and migrated cells are indicated with different shadows between the starting points. B. Graphical representation of the area covered by $\mathrm{H} 4$ cells after $24 \mathrm{~h}$ from three independent experiments. ${ }^{*} \mathrm{p}<0.05,{ }^{* *} \mathrm{p}<0.01,{ }^{* * *} \mathrm{p}<0.005$.

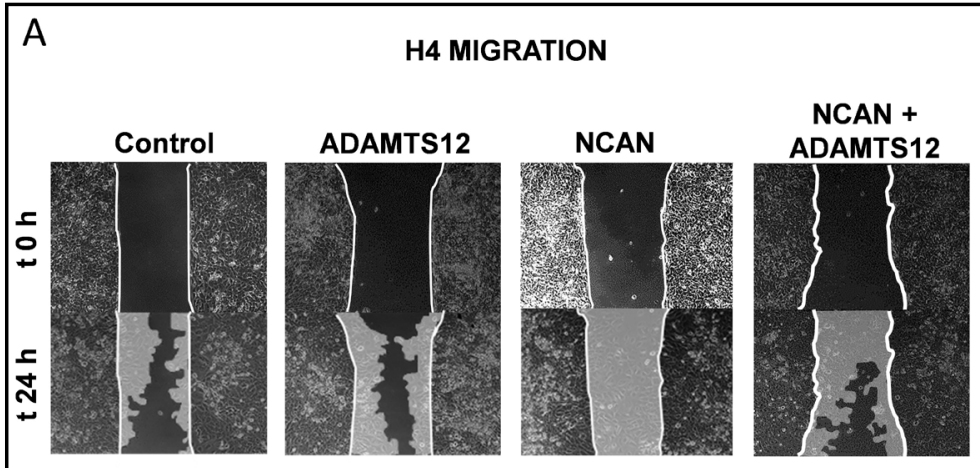

B

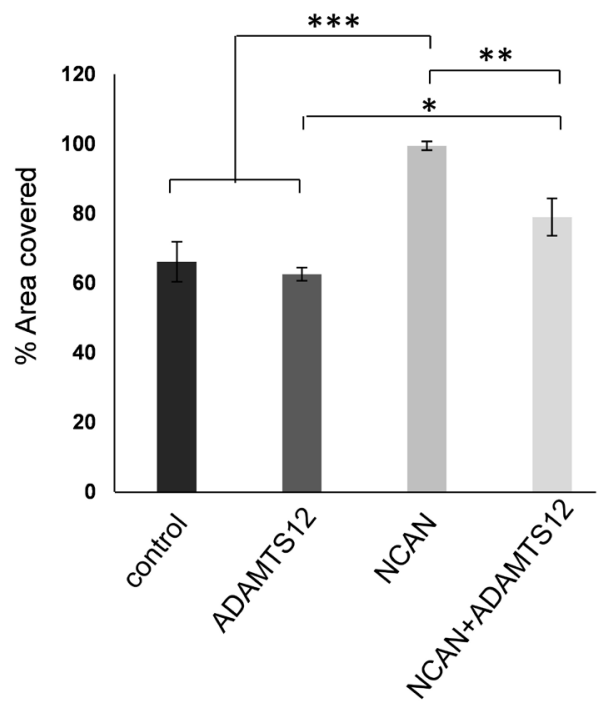




\section{Discussion}

In this work, we found that neurocan is a novel substrate for members of the ADAMTS family, but in particular for ADAMTS12. Consequently, ADAMTS12 can be considered a new hyalectanase, a name employed to depict those metalloproteases able to degrade brevican, aggrecan, versican, or neurocan [24, 25]. Some wellknown hyalectanases include ADAMTS1, ADAMTS4, ADAMTS5, ADAMTS8, ADAMTS9, ADAMTS15, and ADAMTS20 [26, 27]. Taking into account its specificity toward neurocan cleavage, ADAMTS12 could be considered as a "neurocanase," in analogy to the name aggrecanase used to identify ADAMTS4 and ADAMTS5 as aggrecan-cleaving enzymes.

Brevican, versican, and neurocan are involved in the creation of perineuronal nets that regulate complex processes such as synaptic plasticity [28], axonal growth [29], and neuronal migration [30, 31]. Given the importance of hyalectans in brain development, it is expected that there is a mechanism to control their replacement. The most common mechanism, both inside and outside of the nervous system, is proteolysis by metalloproteases such as ADAMTSs [32]. Our data demonstrating that ADAMTS12 is a neurocan-cleaving enzyme opens the possibility to study its role not only in normal conditions but also in different neuropathologies, including those related to alterations of normal behavior. In this regard, both neurocan and ADAMTS12 are components of nervous tissue ECM that have already been linked to certain brain disorders. Neurocan has been associated with schizophrenia and bipolar behavior [33-35], while ADAMTS12 has been genetically linked to cases of schizophrenia in Puerto Rico [21]. In addition, variants of the ADAMTS12 gene are also associated with bipolar disorder [36] and narcolepsy [37] in Japan. Interestingly, our data and analysis of databases related to gene expression in the brain indicate that both neurocan

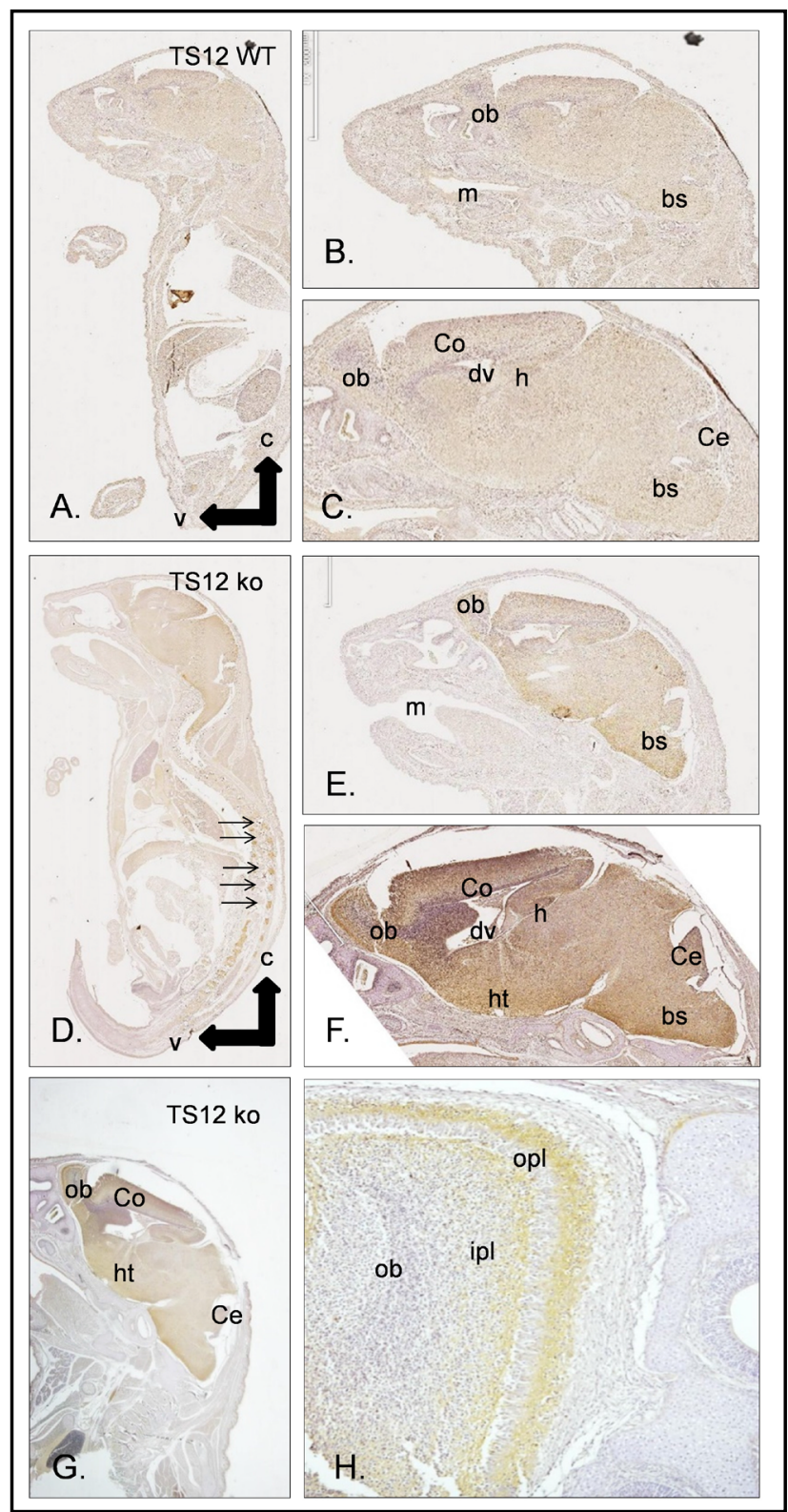

Fig. 5. Immunohistochemical analysis of neurocan expression in neonatal mouse brain. Complete parasagittal sections of a wild-type mouse (wt; A) and an Adamts12-deficient mouse (TS12 ko; D). An immunoreaction was only detected in Adamts12-deficient mice (D-H). m: mouth; ob: olfactory bulb; Ce: cerebellum; Co: cortex; h: hippocampus; ht: hypothalamus; bs: brain stem; dv: diencephalic vector; c: cephalic; v: ventral; opl: outer plexiform layer; ipl: internal plexiform layer. The arrows indicate an immunoreaction for neurocan in the rachidial ganglia of the spinal nerves. 
and ADAMTS12 can be detected in the olfactory bulb in the brain, a region that undergoes functional and morphometric changes in patients with schizophrenia [38].

Our data also showed the high expression levels of neurocan and ADAMTS12 during embryonic development in different parts of the forming nervous system. The embryonic expression of neurocan has been reported [39]. This hyalectan showed a high level of expression at the late stages of embryonic development, whereas it begins to be reduced after birth. The increase of neurocan expression during late embryogenesis may be due to the fact that it plays an important role in brain development, especially in axonal growth and the maintenance of cerebral ECM. But after birth, this function is no longer necessary and the expression of neurocan is reduced slowly, and it is also proteolytically cleaved [16]. The fact that the level of neurocan protein remains elevated in Adamts12-deficient mice after birth provides support for our hypothesis that neurocan is a novel substrate of ADAMTS12. Furthermore, our data also indicate that ADAMTS12 could be the main neurocan-degrading enzyme according to the results obtained for other hyalectanases such as ADAMTS1, ADAMTS4, and ADAMTS5 $[40,41]$ under the same experimental conditions. Therefore, it can be hypothesized that neurocan degradation by ADAMTS12 could be part of a regulated process aimed at removing excessive amounts of neurocan following damage to brain tissue. Consequently, the absence of ADAMTS12 could be an explanation for the accumulation of neurocan in Adamts12-deficient mice, which could affect essential brain functions. In this sense, several studies have established an association between hyalectans and neuronal migration. Neurocan and versican are expressed in the marginal zone and brain subplate, which are well-known areas in relation to neuronal migration [42]. It is also known that neurocan interacts with factors involved in growth and cell motility such as FGF-2, HB-GAM, and amphoterin [14]. Furthermore, neuronal migration favored by HB-GAM can be blocked by the chondroitin 6-sulfate chains contained in the central region of neurocan molecules [43]. Hence, the role of neurocan in cellular motility is a key part of cicatrization, and the relationship between neurocan and ADAMTS12 described in this work could be important in maintaining tissue homeostasis after a traumatic brain injury. This relationship is underlined by the fact that some of the functions requiring the involvement of ADAMTS12 are related to an increase of its expression during inflammation [18, 44].

The relationship between neurocan and ADAMTS12 somewhat resembles that described previously between ADAMTS13 and von Willebrand factor (vWF) in the pathogenesis of thrombotic thrombocytopenic purpura (TTP) [45]. vWF is a high-molecular-weight proteoglycan that participates in platelet aggregation by establishing interactions between their surface and components of the vascular wall. ADAMTS13 cleaves the multimeric chains of $\mathrm{vWF}$, contributing to adequate homeostasis during thrombus formation [46]. Thus, the functional loss of ADAMTS13 in TTP leads to the frequent formation of thrombi due to the accumulation of platelets and ultra-long forms of vWF [47]. We can speculate that when a traumatic brain injury occurs, neurocan levels increase as an orchestrated response to form a three-dimensional net with other components of the ECM that act as the physical support for cells to repair the damage [48]. In our model, the levels of ADAMTS12 are also increased due to the inflammation triggered by injury [18]. Neurocan is necessary for cells to attach to, but it is also essential to eliminate its excess amounts to complete the healing process [7].

\section{Conclusion}

Our results set the possibility that ADAMTS12 is the main enzyme responsible for neurocan cleavage, an activity that may affect cell migration, as we demonstrated for $\mathrm{H} 4$ cells. Further functional studies are necessary to elucidate the precise involvement of ADAMTS12 in brain pathologies through alterations in the cleavage of neurocan. Ongoing projects in our laboratory examining the repair mechanism in wild-type and Adamts12-deficient mice following controlled brain injury will help to shed light on the precise role of neurocan and ADAMTS12 in this process. In addition, it would be very relevant to explore whether 


\section{Cellular Physiology Cell Physiol Biochem 2019;52:1003-1016 \begin{tabular}{ll|l} 
and Biochemistry & $\begin{array}{l}\text { DOl: 10.33594/000000069 } \\
\text { Published online: } 13 \text { April } 2019\end{array}$ & $\begin{array}{l}\text { O } 2019 \text { The Author(s). Published by } \\
\text { Cell Physiol Biochem Press GmbH\&Co. KG }\end{array}$ \\
\cline { 2 - 3 }
\end{tabular} \\ Fontanil et al.: Neurocan Cleavage by ADAMTS12}

Adamts12-deficient mice show any neurological phenotype through the employment of behavioral tests. Meanwhile, our data strongly suggest the importance of ADAMTS12 in the normal maintenance of brain function.

\section{Acknowledgements}

This work was supported by the Instituto Asturiano de Odontología (IAO). Tania Fontanil is a recipient of a contract from the IAO, and Yamina Mohamedi is a recipient of a fellowship from the FICYT (Principado de Asturias). We thank Drs. Gabriel Bretones, Alejandro Piñeiro, Xurde Menéndez, and Jorge Espina for their valuable comments, and we also thank the "Histopatología molecular en modelos animales en cáncer" unit from the IUOPA for the elaboration of the mouse brain samples for immunohistochemistry employed in this work. The study methodologies conformed to the standards set by the Declaration of Helsinki and were approved by the Ethics Committee of the Universidad de Oviedo-Principado de Asturias (Spain).

\section{Disclosure Statement}

The authors declare that no conflicts of interest exist.

\section{References}

1 Barros CS, Franco SJ, Muller U: Extracellular matrix: functions in the nervous system. Cold Spring Harb Perspect Biol 2011;3:a005108.

2 Howell MD, Gottschall PE: Lectican proteoglycans, their cleaving metalloproteinases, and plasticity in the central nervous system extracellular microenvironment. Neuroscience 2012;217:6-18.

3 Dancevic CM, McCulloch DR, Ward AC: The ADAMTS hyalectanase family: biological insights from diverse species. Biochem J 2016;473:2011-2022.

4 Avram S, Shaposhnikov S, Buiu C, Mernea M: Chondroitin sulfate proteoglycans: structurefunction relationship with implication in neural development and brain disorders. Biomed Res Int 2014;2014:642798.

5 Davies SJ, Fitch MT, Memberg SP, Hall AK, Raisman G, Silver J: Regeneration of adult axons in white matter tracts of the central nervous system. Nature 1997;390:680-683.

6 Davies SJ, Goucher DR, Doller C, Silver J: Robust regeneration of adult sensory axons in degenerating white matter of the adult rat spinal cord. J Neurosci 1999;19:5810-5822.

7 Bartus K, James ND, Bosch KD, Bradbury EJ: Chondroitin sulphate proteoglycans: key modulators of spinal cord and brain plasticity. Exp Neurol 2012;235:5-17.

8 Gottschall PE, Howell MD: ADAMTS expression and function in central nervous system injury and disorders. Matrix Biol 2015;44-46:70-76.

9 Cua RC, Lau LW, Keough MB, Midha R, Apte SS, Yong VW: Overcoming neurite-inhibitory chondroitin sulfate proteoglycans in the astrocyte matrix. Glia 2013;61:972-984.

10 Kelwick R, Desanlis I, Wheeler GN, Edwards DR: The ADAMTS (A Disintegrin and Metalloproteinase with Thrombospondin motifs) family. Genome Biol 2015;16:113.

11 Nakada M, Miyamori H, Kita D, Takahashi T, Yamashita J, Sato H, Miura R, Yamaguchi Y, Okada Y: Human glioblastomas overexpress ADAMTS-5 that degrades brevican. Acta Neuropathol 2005;110:239-246.

12 Song RH, Tortorella MD, Malfait AM, Alston JT, Yang Z, Arner EC, Griggs DW: Aggrecan degradation in human articular cartilage explants is mediated by both ADAMTS-4 and ADAMTS-5. Arthritis Rheum 2007;56:575-585.

13 McCulloch DR, Nelson CM, Dixon LJ, Silver DL, Wylie JD, Lindner V, Sasaki T, Cooley MA, Argraves WS, Apte SS: ADAMTS metalloproteases generate active versican fragments that regulate interdigital web regression. Dev Cell 2009;17:687-698. 


\section{Cellular Physiology and Biochemistry}

Cell Physiol Biochem 2019;52:1003-1016

\begin{tabular}{l|l}
\hline DOI: 10.33594/000000069 & (c) 2019 The Author(s). Published by \\
\hline
\end{tabular}

Fontanil et al.: Neurocan Cleavage by ADAMTS12

14 Rauch U, Feng K, Zhou XH: Neurocan: a brain chondroitin sulfate proteoglycan. Cell Mol Life Sci 2001;58:1842-1856.

- 15 Asher RA, Morgenstern DA, Fidler PS, Adcock KH, Oohira A, Braistead JE, Levine JM, Margolis RU, Rogers JH, Fawcett JW: Neurocan is upregulated in injured brain and in cytokine-treated astrocytes. J Neurosci 2000;20:2427-2438.

- 16 Zhou XH, Brakebusch C, Matthies H, Oohashi T, Hirsch E, Moser M, Krug M, Seidenbecher CI, Boeckers TM, Rauch U, Buettner R, Gundelfinger ED, Fassler R: Neurocan is dispensable for brain development. Mol Cell Biol 2001;21:5970-5978.

17 Didangelos A, Puglia M, Iberl M, Sanchez-Bellot C, Roschitzki B, Bradbury EJ: High-throughput proteomics reveal alarmins as amplifiers of tissue pathology and inflammation after spinal cord injury. Sci Rep 2016;6:21607.

18 Moncada-Pazos A, Obaya AJ, Llamazares M, Heljasvaara R, Suarez MF, Colado E, Noel A, Cal S, Lopez-Otin C: ADAMTS-12 metalloprotease is necessary for normal inflammatory response. J Biol Chem 2012;287:3955439563.

19 Llamazares M, Obaya AJ, Moncada-Pazos A, Heljasvaara R, Espada J, Lopez-Otin C, Cal S: The ADAMTS12 metalloproteinase exhibits anti-tumorigenic properties through modulation of the Ras-dependent ERK signalling pathway. J Cell Sci 2007;120:3544-3552.

- 20 Song I, Dityatev A: Crosstalk between glia, extracellular matrix and neurons. Brain Res Bull 2018;136:101108.

- 21 Bespalova IN, Angelo GW, Ritter BP, Hunter J, Reyes-Rabanillo ML, Siever LJ, Silverman JM: Genetic variations in the ADAMTS12 gene are associated with schizophrenia in Puerto Rican patients of Spanish descent. Neuromolecular medicine 2012;14:53-64.

- 22 Colantuoni C, Lipska BK, Ye T, Hyde TM, Tao R, Leek JT, Colantuoni EA, Elkahloun AG, Herman MM, Weinberger DR, Kleinman JE: Temporal dynamics and genetic control of transcription in the human prefrontal cortex. Nature 2011;478:519-523.

- 23 Stanton H, Melrose J, Little CB, Fosang AJ: Proteoglycan degradation by the ADAMTS family of proteinases. Biochim Biophys Acta 2011;1812:1616-1629.

- 24 Schaefer L, Schaefer RM: Proteoglycans: from structural compounds to signaling molecules. Cell Tissue Res 2010;339:237-246.

25 Carulli D, Pizzorusso T, Kwok JC, Putignano E, Poli A, Forostyak S, Andrews MR, Deepa SS, Glant TT, Fawcett JW: Animals lacking link protein have attenuated perineuronal nets and persistent plasticity. Brain 2010;133:2331-2347.

- 26 Rao C, Foernzler D, Loftus SK, Liu S, McPherson JD, Jungers KA, Apte SS, Pavan WJ, Beier DR: A defect in a novel ADAMTS family member is the cause of the belted white-spotting mutation. Development 2003;130:4665-4672.

- 27 Apte SS: A disintegrin-like and metalloprotease (reprolysin type) with thrombospondin type 1 motifs: the ADAMTS family. Int J Biochem Cell Biol 2004;36:981-985.

28 Maeda N, Fukazawa N, Ishii M: Chondroitin sulfate proteoglycans in neural development and plasticity. Front Biosci (Landmark Ed) 2010;15:626-644.

29 Mahmood A, Wu H, Qu C, Mahmood S, Xiong Y, Kaplan DL, Chopp M: Suppression of neurocan and enhancement of axonal density in rats after treatment of traumatic brain injury with scaffolds impregnated with bone marrow stromal cells. J Neurosurg 2014;120:1147-1155.

30 Bandtlow CE, Zimmermann DR: Proteoglycans in the developing brain: new conceptual insights for old proteins. Physiol Rev 2000;80:1267-1290.

31 Berglof E, Plantman S, Johansson S, Stromberg I: Inhibition of proteoglycan synthesis affects neuronal outgrowth and astrocytic migration in organotypic cultures of fetal ventral mesencephalon. J Neurosci Res 2008;86:84-92.

- 32 Levy C, Brooks JM, Chen J, Su J, Fox MA: Cell-specific and developmental expression of lectican-cleaving proteases in mouse hippocampus and neocortex. The Journal of comparative neurology 2015;523:629-648.

- 33 Muhleisen TW, Mattheisen M, Strohmaier J, Degenhardt F, Priebe L, Schultz CC, Breuer R, Meier S, Hoffmann P, Investigators G, Rivandeneira F, Hofman A, Uitterlinden AG, Moebus S, Gieger C, Emeny R, Ladwig KH, Wichmann HE, Schwarz M, Kammerer-Ciernioch J et al.: Association between schizophrenia and common variation in neurocan (NCAN), a genetic risk factor for bipolar disorder. Schizophr Res 2012;138:69-73. 


\section{Cellular Physiology and Biochemistry}

Cell Physiol Biochem 2019;52:1003-1016

\begin{tabular}{l|l}
\hline DOI: 10.33594/000000069 & ( 2019 The Author(s). Published by
\end{tabular}

Fontanil et al.: Neurocan Cleavage by ADAMTS12

34 Oruc L, Kapur-Pojskic L, Ramic J, Pojskic N, Bajrovic K: Assessment of relatedness between neurocan gene as bipolar disorder susceptibility locus and schizophrenia. Bosn J Basic Med Sci 2012;12:245-248.

35 Schultz CC, Muhleisen TW, Nenadic I, Koch K, Wagner G, Schachtzabel C, Siedek F, Nothen MM, Rietschel M, Deufel T, Kiehntopf M, Cichon S, Reichenbach JR, Sauer H, Schlosser RG: Common variation in NCAN, a risk factor for bipolar disorder and schizophrenia, influences local cortical folding in schizophrenia. Psychol Med 2014;44:811-820.

36 Hattori E, Toyota T, Ishitsuka Y, Iwayama Y, Yamada K, Ujike H, Morita Y, Kodama M, Nakata K, Minabe Y, Nakamura K, Iwata Y, Takei N, Mori N, Naitoh H, Yamanouchi Y, Iwata N, Ozaki N, Kato T, Nishikawa T et al.: Preliminary genome-wide association study of bipolar disorder in the Japanese population. Am J Med Genet B Neuropsychiatr Genet 2009;150B:1110-1117.

37 Koike A, Nishida N, Inoue I, Tsuji S, Tokunaga K: Genome-wide association database developed in the Japanese Integrated Database Project. J Hum Genet 2009;54:543-546.

38 Nguyen AD, Pelavin PE, Shenton ME, Chilakamarri P, McCarley RW, Nestor PG, Levitt JJ: Olfactory sulcal depth and olfactory bulb volume in patients with schizophrenia: an MRI study. Brain imaging and behavior 2011;5:252-261.

39 Meyer-Puttlitz B, Junker E, Margolis RU, Margolis RK: Chondroitin sulfate proteoglycans in the developing central nervous system. II. Immunocytochemical localization of neurocan and phosphacan. J Comp Neurol 1996;366:44-54.

40 Miguel RF, Pollak A, Lubec G: Metalloproteinase ADAMTS-1 but not ADAMTS-5 is manifold overexpressed in neurodegenerative disorders as Down syndrome, Alzheimer's and Pick's disease. Brain Res Mol Brain Res 2005;133:1-5.

41 Theocharis AD, Skandalis SS, Tzanakakis GN, Karamanos NK: Proteoglycans in health and disease: novel roles for proteoglycans in malignancy and their pharmacological targeting. FEBS J 2010;277:3904-3923.

42 Maeda N: Proteoglycans and neuronal migration in the cerebral cortex during development and disease. Front Neurosci 2015;9:98.

43 Maeda N, Noda M: Involvement of receptor-like protein tyrosine phosphatase zeta/RPTPbeta and its ligand pleiotrophin/heparin-binding growth-associated molecule (HB-GAM) in neuronal migration. J Cell Biol 1998;142:203-216.

44 Wei J, Richbourgh B, Jia T, Liu C: ADAMTS-12: a multifaced metalloproteinase in arthritis and inflammation. Mediators Inflamm 2014;2014:649718.

45 Sadler JE: Von Willebrand factor, ADAMTS13, and thrombotic thrombocytopenic purpura. Blood 2008;112:11-18.

46 Dong JF, Moake JL, Nolasco L, Bernardo A, Arceneaux W, Shrimpton CN, Schade AJ, McIntire LV, Fujikawa K, Lopez JA: ADAMTS-13 rapidly cleaves newly secreted ultralarge von Willebrand factor multimers on the endothelial surface under flowing conditions. Blood 2002;100:4033-4039.

47 Levy B, Lacolley P, Regnault V: ADAMTS-13 (A disintegrin-like and metalloprotease with thrombospondin) and endothelial dysfunction in sepsis: marker or culprit? Crit Care Med 2007;35:2453-2454.

48 Jones LL, Margolis RU, Tuszynski MH: The chondroitin sulfate proteoglycans neurocan, brevican, phosphacan, and versican are differentially regulated following spinal cord injury. Exp Neurol 2003;182:399-411. 